\title{
Propiedades psicométricas del Cuestionario de Resiliencia para Niños y Adolescentes en estudiantes mexicanos de bachillerato
}

\author{
Héctor Rubén Bravo-Andrade; Fabiola González-Betanzos; Norma Alicia Ruvalcaba-Romero; \\ Judith López-Peñaloza; Mercedes Gabriela Orozco-Solís
}

How to cite this article:

Bravo-Andrade, H.R., González-Betanzos, F., Ruvalcaba-Romero, N.A., López-Peñaloza, J., \& Orozco-Solís, M.G. (2019). Psychometric properties of the Resilience questionnaire for children and adolescents in Mexican Students from high school. Acta Colombiana de Psicología, 22(2), 292-305. doi: http://www.doi.org/10.14718/ACP.2019.22.2.14

Recibido, agosto 6/2018; Concepto de evaluación, octubre 31/2018; Aceptado, marzo 11/2019

\author{
Héctor Rubén Bravo-Andrade* \\ Universidad de Guadalajara, Jalisco, México \\ ORCID: https://orcid.org/0000-0002-0498-8410 \\ Fabiola González-Betanzos \\ Universidad Michoacana de San Nicolás de Hidalgo, Michoacán, México \\ ORCID: https://orcid.org/0000-0003-4585-7211 \\ Norma Alicia Ruvalcaba-Romero \\ Universidad de Guadalajara, Jalisco, México \\ ORCID: https://orcid.org/0000-0001-9209-8751 \\ Judith López-Peñaloza \\ Universidad Michoacana de San Nicolás de Hidalgo, Michoacán, México \\ ORCID: https://orcid.org/0000-0003-0106-3639 \\ Mercedes Gabriela Orozco-Solís \\ Universidad de Guadalajara, Jalisco, México \\ ORCID: https://orcid.org/0000-0002-0648-8233
}

\begin{abstract}
Resumen
El estudio de la resiliencia, capacidad que implica un proceso de adaptación positiva a condiciones de adversidad, ha tenido un creciente interés en los últimos años que demanda la necesidad de contar con instrumentos de medición válidos y confiables que permitan hacer evaluaciones con rigor científico en diferentes tipos de poblaciones. Por esta razón, la presente investigación tuvo como objetivo analizar las propiedades psicométricas del Cuestionario de Resiliencia para Niños y Adolescentes con una muestra de 512 estudiantes de una preparatoria de la Universidad de Guadalajara, México. Inicialmente, se realizó un análisis factorial exploratorio que arrojó una composición de cuatro dimensiones con 18 ítems que explican $61.18 \%$ de la varianza, con un nivel de confiabilidad alto $(\alpha=.90)$; y un análisis factorial confirmatorio con el que se corroboró el modelo, con niveles adecuados de ajuste. Posteriormente, se evaluó la validez convergente del instrumento con la Escala de Autoestima de Rosenberg, donde se obtuvieron correlaciones positivas significativas con las dimensiones de la resiliencia. Y, por último, se valoró la validez divergente con la Escala de Depresión del Centro de Estudios Epidemiológicos (Revisada), donde se encontraron correlaciones significativas negativas. Al final se discuten los resultados y las implicaciones prácticas.

Palabras clave: resiliencia, adolescentes, análisis factorial exploratorio, análisis factorial confirmatorio.
\end{abstract}

\footnotetext{
* Sierra Nevada 950, Puerta 16, Edificio H, 2o. piso, Colonia Independencia, C.P. 44340, Guadalajara, Jalisco, México. Teléfono (52) 33 10585200 extensión 33738, ruben.bravo.a@academicos.udg.mx

Se agradece a la Dra. Norma Ivonne González-Arratia López Fuentes, autora del Cuestionario de resiliencia para niños y adolescentes por su generosidad al proporcionar el instrumento para investigación. Al Consejo Nacional de Ciencia y Tecnología de México por la beca de manutención $\mathrm{N}^{\circ} 375718$ para estudios de doctorado del primer autor, de donde surge el presente estudio.
} 


\title{
Psychometric properties of the Resilience Questionnaire for children and adolescents in Mexican high school students
}

Abstract

\begin{abstract}
Resilience implies a process of positive adjustment to adverse conditions. The growing interest in their study demands the need for valid and reliable measurement instruments that allow assessments with scientific rigor in different types of populations. The aim of the present research was to analyze the psychometric properties of the Resilience Questionnaire for Children and Adolescents. 512 students from a high school of the University of Guadalajara, Mexico, participated. Exploratory factor analysis yielded a four-dimensional composition with 18 items than explain $61.18 \%$ of the variance, with an excellent level of reliability $(\alpha=.90)$. Through confirmatory factor analysis, the model was corroborated, presenting adequate levels of adjustment. Convergent validity was assessed using Rosenberg's Self-esteem Scale, obtaining significant positive correlations with the dimensions of resilience. The divergent validity was assessed through the Depression Scale of the Epidemiological Studies Center - Revised, showing significant negative correlations. Results and practical implications are discussed.
\end{abstract}

Key words: resilience, adolescents, exploratory factor analysis, confirmatory factor analysis.

\section{Propriedades psicométricas do Questionário de Resiliência para Crianças e Adolescentes em estudantes mexicanos do Ensino Médio}

\author{
Resumo
}

\begin{abstract}
O estudo da resiliência, capacidade que implica um processo de adaptação positiva às condições de adversidade, passou por um crescente interesse nos últimos anos que demanda a necessidade de contar com instrumentos de medição válidos e confiáveis que permitam realizar avaliações com rigor científico em diferentes tipos de população. Por esse motivo, a presente pesquisa teve como objetivo analisar as propriedades psicométricas do Questionário de Resiliência para Crianças e Adolescentes com uma amostra de 512 estudantes do Ensino Médio preparatório da Universidade de Guadalajara, no México. Inicialmente, realizou-se uma análise fatorial exploratória que resultou em uma composição de quatro dimensões com 18 itens que explicam $61,18 \%$ da variância, com um nível de confiabilidade alto $(\alpha=.90)$, e uma análise fatorial confirmatória com a qual se corroborou o modelo, com níveis adequados de ajuste. Posteriormente, avaliou-se a validade convergente do instrumento com a Escala de Autoestima de Rosenberg, com a qual foram obtidas correlações positivas significativas com as dimensões da resiliência. E, por último, aplicou-se a validade divergente com a Escala de Depressão do Centro de Estudos Epidemiológicos (Revisada), com a qual foram encontradas correlações negativas significativas. Por fim, discutem-se os resultados e as implicações práticas.

Palavras-chave: resiliência, adolescentes, análise fatorial exploratória, análise fatorial confirmatória.
\end{abstract}

\section{Introducción}

El campo de estudio de la resiliencia humana se remonta a los años 70 y tiene sus orígenes en la psicología clínica, específicamente en investigaciones sobre el ajuste positivo a condiciones de riesgo para el desarrollo infantil (Masten, 2018); y últimamente ha encontrado un nicho fértil en la psicología positiva, cuyo interés se centra más en las potencialidades que en el déficit o la patología (Belykh, 2018).

Sin embargo, a pesar del creciente interés por dicho constructo, no existe consenso sobre su definición ni sobre los factores que la componen o la manera de medirla (Luthar, 2015; Luthar \& Cushing, 2002). Con respecto al concepto, es posible identificar tres aspectos de convergencia entre las principales definiciones: (a) la noción de adversidad, que refiere a la presencia de uno o más factores de riesgo acumulados, o a la existencia de una calamidad inesperada; (b) la referencia a una adaptación positiva ante dicha adversidad, que no solo implica enfrentarla, sino fortalecerse a partir de ella; y (c) la concepción de la resiliencia como un proceso más que como una característica inherente de la persona (Masten, 2018; Rutter, 2012).

En cuanto al último punto, se pasó de una primera generación de estudios que consideraban la resiliencia como un rasgo distintivo de la persona, a entenderla como un proceso (García-Vesga \& Domínguez de la Ossa, 2013) en el que entran en interacción factores individuales, familiares y sociales (Luthar, 2015).

En el plano individual, las principales características asociadas a la resiliencia son un cociente intelectual alto, estilos de afrontamiento orientados a tareas y solución de problemas, autogestión, locus de control interno, habilidades sociales, empatía, planificación, humor, autoestima, compromiso, y capacidad de reflexión o introspección (Luthar, 2015; Rutter, 
2012; Walsh, 2004). De igual modo, a nivel individual se han considerado aspectos biológicos, como las respuestas fisiológicas involucradas con adaptaciones resilientes (Monroy \& Palacios, 2011). Por otra parte, en el ámbito familiar destacan los sistemas compartidos de creencias, los procesos de organización, la comunicación y la solución de problemas (Walsh, 2004). Y, por último, desde el ámbito social se han tenido en cuenta las redes sociales de apoyo y la cohesión social como soporte de la resiliencia (Luthar, 2015).

Ahora bien, la evaluación de la resiliencia y de sus dimensiones es esencial para el estudio del fenómeno, y en un sentido, Luthar y Cushing (2002) señalan que en la medición usualmente se destaca al menos uno de los elementos mencionados, es decir, que los instrumentos se dirigen al estudio de la resiliencia como la superación de la adversidad, como forma de adaptación, o bien, como proceso. En particular, en las valoraciones sobre la adversidad se estima el riesgo ante múltiples factores de vida específicos; mientras que en la medición de la adaptación positiva se consideran los ajustes en etapas de desarrollo o ausencia de patología; y en la valoración de la resiliencia como proceso se realizan evaluaciones por medio de análisis estadísticos sobre la relación de las variables de riesgo y la adaptación positiva, o al hacer una distinción entre los sujetos resilientes de aquellos que no lo son (Luthar \& Cushing, 2002).

Al respecto, Windle, Bennet y Noyes (2011) analizaron los 15 instrumentos de medición de resiliencia más reportados en la literatura, y, del total de instrumentos analizados, siete de ellos se encuentran dirigidos a población joven o adolescente y uno a población infantil. En la Tabla 1 se describen brevemente dichos instrumentos, junto con los factores que evalúan.

Por otra parte, en la literatura especializada disponible en español se encuentran investigaciones que sostienen que un mayor grado de resiliencia: (a) conlleva a la superación de eventos adversos de vida (Barcelata, Durán \& GómezMaqueo, 2012); (b) funge como una variable protectora ante el suicidio (Quiceno, Mateus, Cárdenas, Villareal \& Vinaccia, 2013; Sánchez-Teruel \& Robles-Bello, 2014; VillalobosGalvis, Arévalo \& Rojas, 2012); (c) guarda una relación positiva significativa con la autoestima (González-Arratia,

Tabla 1.

Descripción de instrumentos que evalúan resiliencia en jóvenes y niños

\begin{tabular}{|c|c|c|c|}
\hline Nombre del instrumento & Autores (año) & $\begin{array}{l}\text { Número de } \\
\text { dimensiones } \\
\text { (ítems) }\end{array}$ & Factores \\
\hline $\begin{array}{l}\text { Youth Resiliency Assessing } \\
\text { Developmental Strengths } \\
\text { (YR:ADS) }\end{array}$ & $\begin{array}{l}\text { Donnon \& } \\
\text { Hammond } \\
\text { (2007) }\end{array}$ & $10(94)$ & $\begin{array}{l}\text { Apoyo paterno/expectativas de cuidado familiar; relación con } \\
\text { pares; cohesión comunitaria; compromiso con el aprendizaje; } \\
\text { cultura escolar; sensibilidad cultural; autocontrol; empodera- } \\
\text { miento; autoconcepto; sensibilidad social. }\end{array}$ \\
\hline $\begin{array}{l}\text { The Connor-Davidson Re- } \\
\text { silience Scale (CD-RISC) }\end{array}$ & $\begin{array}{l}\text { Campbell-Sills } \\
\& \text { Stein }(2007)\end{array}$ & $1(10)$ & Habilidad para enfrentar el estrés. \\
\hline $\begin{array}{l}\text { The Resiliency Attitudes } \\
\text { and Skills Profile }\end{array}$ & $\begin{array}{l}\text { Hurtes \& Allen } \\
(2001)\end{array}$ & $7(34)$ & $\begin{array}{l}\text { Insight, independencia, creatividad, humor, iniciativa, relacio- } \\
\text { nes, orientación a valores. }\end{array}$ \\
\hline Adolescent Resilience Scale & $\begin{array}{c}\text { Oshio, } \\
\text { Kaneko, } \\
\text { Nagamine \& } \\
\text { Nakaya (2003) } \\
\end{array}$ & $3(21)$ & $\begin{array}{l}\text { Búsqueda de novedad, regulación emocional, orientación posi- } \\
\text { tiva hacia el futuro. }\end{array}$ \\
\hline $\begin{array}{l}\text { The Child and Youth Resil- } \\
\text { ience Measure (CYRM) }\end{array}$ & $\begin{array}{l}\text { Ungar et al. } \\
(2008)\end{array}$ & $4(28)$ & Dominio individual, relacional, comunitario y cultural. \\
\hline $\begin{array}{l}\text { Resilience Scale for Adoles- } \\
\text { cents (READ) }\end{array}$ & $\begin{array}{l}\text { Hjmedal, } \\
\text { Friborg, Stiles, } \\
\text { Martinussen } \\
\text { \& Rosenvinge } \\
\quad(2006) \\
\end{array}$ & $5(39)$ & $\begin{array}{l}\text { Competencia personal, competencia social, estilo estructurado, } \\
\text { cohesión familiar y recursos sociales. }\end{array}$ \\
\hline The Ego Resilience (ER) & $\begin{array}{c}\text { Bromley, } \\
\text { Johnson \& } \\
\text { Cohen }(2006) \\
\end{array}$ & $4(102)$ & $\begin{array}{l}\text { Optimismo, actividad productiva, insight y calidez, expresivi- } \\
\text { dad hábil. }\end{array}$ \\
\hline $\begin{array}{l}\text { California Healthy Kids } \\
\text { Survey - The Resilience } \\
\text { Scale of the Student Survey }\end{array}$ & $\begin{array}{l}\text { Sun \& Steward } \\
\qquad(2007)\end{array}$ & $12(34)$ & $\begin{array}{l}\text { Comunicación y cooperación, autoestima, empatía, resolu- } \\
\text { ción de problemas, metas y aspiraciones, conexión familiar, } \\
\text { conexión escolar, conexión comunitaria, experiencia autónoma, } \\
\text { conducta prosocial con pares, participación significativa en } \\
\text { actividades de la comunidad, y apoyo de pares. }\end{array}$ \\
\hline
\end{tabular}

Nota. Resumen elaborado a partir de Windle et al. (2011). 
Reyes, Valdéz \& González, 2011); (d) puede ser favorecida en el ámbito escolar mediante actividades significativas que involucren a los adolescentes (Arón \& Milicic, 2011); y (e) se asocia a un nivel menor de depresión (RestrepoRestrepo, Vinaccia \& Quiceno, 2011). Asimismo, se ha encontrado que promover recursos resilientes desde la familia y la escuela protege del embarazo no deseado y del consumo de drogas a adolescentes mayas (Aguiar \& AcleTomasini, 2012), y que programas dirigidos a desarrollar la resiliencia amortiguan los efectos de la pobreza y el estrés (Fiorentino, 2008).

En México, en particular, existen tres instrumentos de resiliencia dirigidos a niños y jóvenes: la Escala de Resiliencia Mexicana (RESI-M; Palomar \& Gómez, 2010), la Escala de Resiliencia para Adolescentes (READ; RuvalcabaRomero, Gallegos-Guajardo \& Villegas-Guinea, 2014), y el Cuestionario de Resiliencia para Niños y Adolescentes (González-Arratia, 2016).

Primero, la Escala de Resiliencia Mexicana (RESI-M) es la adaptación en población mexicana de dos instrumentos publicados originalmente en idioma inglés: The Connor-Davidson Resilience Scale (CD-RISC; Connor \& Davidson, 2003) y The Resilience Scale for Adults (RSA; Friborg, Hjemdal, Rosenvinge \& Martinussen, 2001). Específicamente, esta prueba está dirigida a población de entre 18 y 25 años; su análisis señala un nivel de confiabilidad alto $(\alpha=.93)$; y mediante el análisis de componentes principales con rotación varimax la prueba obtuvo una estructura factorial de cinco dimensiones que explican el $43.6 \%$ de la varianza: fortaleza y confianza en sí mismo, de 19 reactivos $(\alpha=.92)$; competencia social, de 8 ítems $(\alpha=.87)$; apoyo familiar, de 6 reactivos $(\alpha=.87)$; apoyo social, con 5 ítems $(\alpha=.84)$; y estructura, con 5 reactivos $(\alpha=.79)$ (Palomar \& Gómez, 2010). Este instrumento se ha utilizado en población adolescente para analizar la relación entre resiliencia y la felicidad frente a la marginación urbana en México, a partir de lo cual se ha encontrado una relación negativa entre la marginación y la resiliencia, así como una relación positiva entre resiliencia y felicidad (Gómez-Azcarate et al., 2014).

Segundo, la Escala de Resiliencia para Adolescentes surge de la adaptación del instrumento The Resilience Scale for Adolescents (READ; Hjemdal et al., 2006), y su adaptación en población mexicana se realizó en adolescentes de entre 12 a 17 años por medio del método de componentes principales, donde se encontró una estructura de cinco dimensiones con 22 reactivos. En esta adaptación se conservaron cuatro de los factores originales de la escala: cohesión familiar $(\alpha=.82)$, competencia social $(\alpha=.73)$, competencia personal $(\alpha=.64)$ y recursos sociales $(\alpha=.70)$, mientras que la quinta dimensión, estilo estructurado, fue eliminada, pero en el proceso de validación los autores añadieron un factor denominado orientación a metas $(\alpha=.60)$ (Ruvalcaba-Romero et al., 2014). Cabe mencionar que para la adaptación se reportaron correlaciones significativas con competencias socioemocionales y autoestima, así como correlaciones negativas significativas con ansiedad y depresión (Ruvalcaba-Romero et al., 2014). El READ para población mexicana se utilizó en un estudio sobre prácticas parentales, donde se encontró que, a mayor autonomía, comunicación y control conductual parental, mayor grado de resiliencia (Ruvalcaba-Romero, Gallegos-Guajardo, Caballo \& Villegas-Guinea, 2016).

Y tercero, el Cuestionario de Resiliencia para Niños y Adolescentes es un instrumento desarrollado y estudiado con población mexicana, en el cual, por medio de un análisis factorial de componentes principales con rotación varimax, se obtuvieron tres dimensiones que explican el $37.82 \%$ de la varianza: (a) factores protectores internos $(\alpha=.80)$, que evalúan capacidades interpersonales y de resolución de problemas; (b) factores protectores externos $(\alpha=.73)$, que indican el apoyo familiar percibido por el adolescente; y (c) empatía ( $\alpha=.78$ ), que valora el comportamiento altruista y prosocial. El alfa de Cronbach total reportado es de .91 (González-Arratia et al., 2011). Este cuestionario se encuentra sustentado bajo el modelo teórico de Grotberg (2006) y ha sido utilizado para estudiar la relación entre resiliencia y diversas variables, como autoestima, personalidad resistente, vínculo parental, estilos de afrontamientos, locus de control y espiritualidad (González-Arratia, 2016); además del estudio del capital psicológico en relación con el estilo de vida saludable (González-Cantero et al., 2017); y la relación entre resiliencia y optimismo con la escala total $(\alpha=.88)$ y cada una de sus dimensiones: factores protectores internos $(\alpha=80)$, factores protectores externos $(\alpha=.73)$ y empatía $(\alpha=.78)$ (González \& Valdez, 2012). A pesar de que se han hecho diversos estudios con este instrumento, hasta el momento no se ha informado sobre la dimensionalidad de la escala por medio del análisis factorial confirmatorio, ni se han encontrado estudios que comparen el ajuste de diferentes modelos.

Entre las principales limitaciones en la medición de la resiliencia destaca la falta de un estándar; que muchas de las escalas requieren métodos de validación más robustos; que el sentido del constructo puede tener un componente cultural y contextual (Windle et al., 2011); y que el no contar con instrumentos válidos y confiables dificulta las comparaciones con rigor científico (Luthar \& Cushing, 2002). Teniendo esto en cuenta, el objetivo del presente estudio fue analizar las propiedades psicométricas del Cuestionario de Resiliencia para Niños y Adolescentes en una muestra de estudiantes de preparatoria. 
296

Método

\section{Tipo de estudio}

Se realizó un estudio descriptivo, transversal, que consistió en la medición de variables en un momento único, en un tiempo determinado (Álvarez, González \& Chávez, 2017).

\section{Participantes}

En total, participaron 512 estudiantes de una escuela pública urbana de educación media superior de Guadalajara, México, de los cuales el $55.7 \%$ (285) eran mujeres y el $44.3 \%$ (227), hombres. El promedio de edad fue de 15 años $(\mathrm{DE}=0.54)$, con un rango de 14 a 18 años. Los participantes fueron elegidos de manera no probabilística, mediante un muestreo propositivo.

\section{Instrumentos}

Cuestionario de resiliencia para niños y adolescentes. Este cuestionario, elaborado por González-Arratia (2016), se compone de 32 ítems -con opciones de respuesta tipo Likert, de $1=$ nunca a $5=$ siempre-, organizados en tres dimensiones: factores protectores externos, factores protectores internos y empatía. El resto de las características y propiedades del instrumento fueron previamente descritos en el apartado de la introducción.

Escala de Autoestima de Rosenberg. Este instrumento fue validado en una población de 100 adolescentes mexicanos de entre 13 y 15 años de edad, con una varianza explicada del 66.2 \% (González-Forteza \& Rodríguez, 1993). La escala está compuesta por 10 reactivos que evalúan pensamientos y sentimientos de valía personal y de respeto a sí mismo, con un formato de respuesta de 1 = "totalmente de acuerdo" a 4 = "totalmente en desacuerdo". Los reactivos 1, 2, 4, 6 y 7 se recodifican para obtener un rango teórico de 10 a 40, donde a mayor puntaje, mayor nivel de autoestima. Este instrumento, en su adaptación de Jiménez, Mondragón y González-Forteza (2007) en población adolescente mexicana, ha reportado un nivel de consistencia interna aceptable, con un alfa de Cronbach de .68 a .78, lo cual coincide con los resultados de la presente investigación.

Escala de Depresión del Centro de Estudios Epidemiológico (Revisada) (CES-D-R). Esta prueba, validada por González-Forteza, Jiménez-Tapia, Ramos-Lira y Wagner (2008), consta de 35 reactivos que indican la presencia en las últimas dos semanas de síntomas depresivos, a partir de una escala de tipo Likert con opciones de respuesta: 0 $=0$ días, $1=1-2$ días, $2=3-4$ días, $3=5-7$ días, y $4=8-14$ días. En una muestra de 1549 estudiantes con media de edad de 14 años $(\mathrm{DE}=1.2)$ se realizó un análisis factorial con extracción de componentes principales y rotación oblicua, donde se identificaron seis factores que explican el $54.61 \%$ de la varianza: afecto deprimido ( $\alpha=.90 ; 5$ reactivos), afecto positivo ( $\alpha=.80 ; 3$ ítems), inseguridad emocional y problemas interpersonales ( $\alpha=.94 ; 10$ reactivos), somatización ( $\alpha=.91 ; 7$ ítems), bienestar emocional $(\alpha=.81$; 3 reactivos) y actividad retardada ( $\alpha=.92 ; 6$ ítems). En la presente investigación, los niveles de confiablidad -en el orden de aparición antes descrito- fueron: .86, .83, .88, $.83, .79, .76$, todos en niveles aceptables.

\section{Procedimiento}

Para la administración de los instrumentos se llevó a cabo un procedimiento online mediante grupos intactos constituidos por los grupos de clase. En particular, las pruebas fueron aplicadas por medio de Google Forms en los laboratorios de cómputo de la escuela durante el horario regular de clases. Una vez llevada a cabo la instrucción para contestar los instrumentos -en un tiempo promedio de respuesta de 20 minutos-, se procedió a aclarar dudas. Cabe mencionar que se tuvo en cuenta a todos los estudiantes de primer ingreso, a quienes se les solicitó la firma de la carta de consentimiento informado por parte de sus padres o tutores para otorgar el permiso para participar de manera voluntaria en la investigación; en dicha carta se informó sobre la confidencialidad de los datos. Este estudio fue avalado por el comité de ética y las autoridades de la escuela, y se atuvo a los lineamientos éticos nacionales e internacionales.

\section{Análisis de datos}

Con el fin de evitar la capitalización del azar, se dividieron los datos de manera aleatoria en dos muestras aproximadamente iguales, una para el análisis factorial exploratorio $(\mathrm{n}=251)$-con el programa SPSS, versión 23-, y otra para el análisis factorial confirmatorio $(\mathrm{n}=261)$-mediante el programa AMOS, versión 16-.

Inicialmente, para el análisis de los reactivos se establecieron medias, desviaciones estándar, asimetría y curtosis, así como el estadístico de la prueba de KolmogorovSmirnov, junto con el nivel de significación, para evaluar la normalidad de la distribución. Después, se llevó a cabo un análisis factorial exploratorio (AFE) mediante el método de componentes principales con rotación ortogonal varimax, basado en autovalores superiores a 1, donde se tomaron en 
consideración las medidas de Kaiser-Meyer-Olkin (KMO) mayores a .80 y la prueba de esfericidad de Bartlett en un nivel significativo.

Los indicadores utilizados para el análisis factorial confirmatorio (AFC) fueron los índices de ajuste absoluto -índice de bondad de ajuste (GFI) e índice de bondad de ajuste corregido (AGFI)-, cuyos valores entre .90 o .95 reflejan un buen modelo; la raíz media cuadrada del error de aproximación (RMSEA), en la que puntuaciones entre .05 y .08 reflejan un ajuste razonable (Schumacker \& Lomax, 2010); y la raíz cuadrada residual (RMR), preferentemente menor a .05 (Escobedo, Hernández, Estebané \& Martínez, 2016). Además de esto, los índices de ajuste incremental considerados fueron el índice de ajuste de normalidad (NFI); el índice de ajuste de Tucker y Lewis (TLI), cuyos valores entre .90 y .95 indican un buen ajuste (Schumacker \& Lomax, 2010); el índice de ajuste comparativo (CFI) mayor a .95; y el índice de ajuste incremental (IFI), cercano a .95. También, se tuvieron en cuenta los criterios de información Akaike (AIC) y bayesiano (BIC) para comparar modelos con distintos factores, donde los valores menores indican un mejor ajuste (Kline, 2011).

Por último, se efectuó el cálculo del coeficiente de confiabilidad de las escalas y sus dimensiones por medio del indicador alfa de Cronbach; y para la evaluación de la validez convergente y divergente se ejecutó la prueba de correlación de Spearman entre las dimensiones de la resiliencia y las escalas de autoestima y depresión, constructos con los que guarda empíricamente una relación (González-Arratia et al., 2011; Restrepo-Restrepo et al., 2011).

\section{Resultados}

En el presente apartado se muestra la factorización del cuestionario, seguida del ajuste del modelo obtenido, las medidas de confiabilidad por dimensión, y los indicadores de validez convergente y divergente.

\section{Análisis factorial exploratorio}

La muestra constituida para el AFE quedó conformada por 251 participantes, con un $57.8 \%$ (145) de mujeres y un $42.2 \%$ (106) de hombres. El promedio de edad fue de 14.99 años $(\mathrm{DE}=0.529)$, con un mínimo de $14 \mathrm{y}$ un máximo de 18.

Con el fin de evaluar normalidad de la distribución, se aplicó la prueba de Kolmogorov-Smirnov, de la cual resultó (véase Tabla 2) que los reactivos no cumplían con tal supuesto. Sin embargo, se tomaron como criterio los parámetros de asimetría \pm 2 y curtosis \pm 7 en los que se puede considerar un comportamiento semejante al normal
(Abad, Olea, Ponsoda \& García, 2011; Curran, West \& Finch, 1996). Los ítems que superaron estos valores -reactivos $12,15,16,17,31$ y $32-$ se descartaron debido a que causaban errores de especificación en los modelos. No se encontraron valores perdidos.

Tomando en consideración que no se encontró normalidad en la distribución de los ítems, se decidió utilizar el método de componentes principales, considerado como el más apropiado para estos casos (Abad et al., 2011). El procedimiento de rotación elegido fue el ortogonal varimax, con el fin de que coincidiera con el utilizado en la escala original (González-Arratia, 2016).

Tabla 2.

Estadísticos descriptivos de los reactivos del Cuestionario de Resiliencia para Niños y Adolescentes $(n=251)$

\begin{tabular}{ccccccc}
\hline N. $^{\circ}$ & Media & DE & K-S & $p$ & Asimetría & Curtosis \\
\hline 1 & 4.52 & 0.712 & .364 & $<.001$ & -1.674 & 2.999 \\
2 & 4.37 & 0.812 & .309 & $<.001$ & -1.509 & 2.588 \\
3 & 4.29 & 0.788 & .263 & $<.001$ & -1.149 & 1.227 \\
4 & 4.31 & 0.842 & .281 & $<.001$ & -1.440 & 2.438 \\
5 & 4.38 & 0.897 & .344 & $<.001$ & -1.568 & 2.158 \\
6 & 4.22 & 0.731 & .280 & $<.001$ & -1.179 & 2.874 \\
7 & 4.12 & 0.857 & .283 & $<.001$ & -1.070 & 1.226 \\
8 & 4.35 & 0.725 & .283 & $<.001$ & -1.164 & 1.587 \\
9 & 4.29 & 0.757 & .257 & $<.001$ & -1.089 & 1.277 \\
10 & 4.45 & 0.780 & .355 & $<.001$ & -1.376 & 1.343 \\
11 & 4.15 & 0.843 & .239 & $<.001$ & -.851 & .426 \\
12 & 4.54 & 0.786 & .377 & $<.001$ & $\mathbf{- 2 . 2 8 5}$ & 6.019 \\
13 & 4.30 & 1.002 & .322 & $<.001$ & -1.551 & 1.754 \\
14 & 4.50 & 0.869 & .392 & $<.001$ & -1.930 & 3.290 \\
15 & 4.81 & 0.501 & .493 & $<.001$ & $\mathbf{- 3 . 2 0 8}$ & $\mathbf{1 2 . 0 9 7}$ \\
16 & 4.80 & 0.496 & .486 & $<.001$ & $\mathbf{- 3 . 5 4 7}$ & $\mathbf{1 7 . 9 2 3}$ \\
17 & 4.71 & 0.534 & .449 & $<.001$ & $\mathbf{- 2 . 0 2 8}$ & 4.967 \\
18 & 4.56 & 0.692 & .386 & $<.001$ & -1.864 & 4.312 \\
19 & 4.14 & 0.852 & .269 & $<.001$ & -.929 & .443 \\
20 & 4.25 & 0.797 & .262 & $<.001$ & -.904 & .370 \\
21 & 4.22 & 0.811 & .268 & $<.001$ & -1.139 & 1.469 \\
22 & 4.12 & 0.840 & .288 & $<.001$ & -.964 & .654 \\
23 & 4.16 & 0.811 & .265 & $<.001$ & $\mathbf{- . 8 9 8}$ & .549 \\
24 & 3.95 & 0.968 & .258 & $<.001$ & -.749 & -.106 \\
25 & 4.31 & 0.823 & .270 & $<.001$ & -1.404 & 2.081 \\
26 & 4.29 & 0.885 & .279 & $<.001$ & -1.443 & 1.910 \\
27 & 4.41 & 0.700 & .304 & $<.001$ & -1.248 & 1.950 \\
28 & 4.29 & 0.880 & .280 & $<.001$ & -1.455 & 2.156 \\
29 & 4.24 & 0.889 & .262 & $<.001$ & -1.280 & 1.426 \\
30 & 4.55 & 0.675 & .387 & $<.001$ & -1.532 & 2.184 \\
31 & 4.63 & 0.682 & .418 & $<.001$ & $\mathbf{- 2 . 4 3 8}$ & $\mathbf{7 . 6 1 8}$ \\
32 & 4.57 & 0.719 & .393 & $<.001$ & $\mathbf{- 2 . 1 4 5}$ & 5.772 \\
\hline & & & & & &
\end{tabular}


Una vez eliminados los reactivos que no cumplían con el supuesto de normalidad, se procedió a realizar el estudio exploratorio. Antes de llegar al modelo final, se evaluaron dos modelos previos: en el primero resultaron cinco dimensiones que explicaban el $61 \%$ de la varianza $(\mathrm{KMO}=.91, \mathrm{p}<.001$, comunalidades $>.50, \mathrm{X}^{2}=3302.206, \mathrm{gl}=325$ ), pero en búsqueda del modelo con mejores indicadores de ajuste, $\mathrm{y}$ atendiendo a las consideraciones planteadas por Méndez y Rondón (2012), se decidió eliminar los reactivos 30, 18,9 y 23 , debido a que contaban con cargas factoriales menores a .50 , consideradas de aporte mínimo; y del segundo modelo resultante, de cinco dimensiones que explicaban el $63.69 \%$ de la varianza $(\mathrm{KMO}=.90, \mathrm{p}<.001$, comunalidades $>.50$, $\mathrm{X}^{2}=2616.996, \mathrm{gl}=231$ ), se eliminaron los ítems 28 y 5 por la razón antes descrita, junto a los reactivos 22 y 19 por no alcanzar el criterio de un mínimo de tres elementos para conformar una dimensión.

Mediante la depuración de reactivos resultó un modelo factorial final con un índice $\mathrm{KMO}=.90 \mathrm{y}$ una prueba de esfericidad de Bartlett significativa $\left(X^{2}=1879.924\right.$, $\mathrm{gl}=153, \mathrm{p}<.001)$, con comunalidades superiores a .50 , que conforman cuatro dimensiones que explican el $61.18 \%$ de la varianza. Los autovalores encontrados van de 6.8 a
1. La carga factorial menor de los reactivos fue de .50 , y la mayor, de .82 .

Dado que los factores 1 y 3 mostraron una correlación alta, se decidió llevar a cabo un AFE con los ítems involucrados. Mediante los resultados se corroboró que se trata de dos factores diferenciados $(\mathrm{KMO}=.908$; Bartlett <.001; comunalidades > .40; autovalores: componente $1=4.69$, componente $2=1.14$; varianza explicada del $58.23 \%$ ), cuyos ítems se distribuyen de la misma manera que en el análisis previo.

En la Tabla 3 se aprecia que los cinco ítems que forman el primer componente son parte de la dimensión llamada "factores protectores internos" de la escala original, la cual se describe como capacidades interpersonales y habilidades de resolución de conflictos. Se decidió renombrar el factor bajo el nombre "introspección" para distinguirlo de la tercera dimensión obtenida en el presente análisis.

Los cinco reactivos de la segunda dimensión corresponden al factor "empatía" de la escala original, con excepción del reactivo 1 ("Yo soy feliz cuando hago algo bueno para los demás"), que en el instrumento original formaba parte de los factores protectores externos. De cualquier forma,

Tabla 3.

Matriz de estructura factorial obtenida para el Cuestionario de Resiliencia para Niños y Adolescentes

\begin{tabular}{|c|c|c|c|c|c|}
\hline & \multirow{2}{*}{ Reactivo } & \multicolumn{4}{|c|}{ Factor } \\
\hline & & 1 & 2 & 3 & 4 \\
\hline RE26 & Puedo imaginar las consecuencias de mis actos. & .77 & & & \\
\hline RE25 & Puedo buscar la manera de resolver mis problemas. & .75 & & & \\
\hline RE24 & Yo puedo controlar mi vida. & .68 & & & \\
\hline RE27 & Puedo reconocer lo bueno y lo malo para mi vida. & .66 & & & \\
\hline RE29 & Puedo cambiar cuando me equivoco. & .50 & & & \\
\hline RE8 & Soy amable. & & .74 & & \\
\hline RE2 & Yo soy respetuoso de mí mismo y de los demás. & & .71 & & \\
\hline RE1 & Yo soy feliz cuando hago algo bueno para los demás. & & .66 & & \\
\hline RE3 & Soy agradable con mis familiares. & & .66 & & \\
\hline RE7 & Soy acomedido y cooperador. & & .60 & & \\
\hline RE6 & Soy inteligente. & & & .68 & \\
\hline RE21 & Me siento preparado para resolver mis problemas. & & & .64 & \\
\hline RE4 & Soy capaz de hacer lo que quiero. & & & .63 & \\
\hline RE11 & Conmigo hay personas que quieren que aprenda a desenvolverme solo. & & & .63 & \\
\hline RE20 & Soy firme en mis decisiones. & & & .55 & \\
\hline RE14 & Tengo personas que me quieren a pesar de lo que sea o haga. & & & & .82 \\
\hline RE13 & Cerca de mí hay amigos en quien confiar. & & & & .77 \\
\hline RE10 & Yo tengo personas que me quieren incondicionalmente. & & & & .66 \\
\hline & N. ${ }^{\circ}$ de reactivos & 5 & 5 & 5 & 3 \\
\hline & Porcentaje de varianza explicada & 17.4 & 16.4 & 14.6 & 12.6 \\
\hline & Autovalores & 6.8 & 1.6 & 1.4 & 1 \\
\hline
\end{tabular}

Nota: Factor 1 = introspección; Factor 2 = empatía; Factor 3 = resolución de problemas; Factor 4 = factores protectores externos. Fuente: elaboración propia. 
se decidió conservarlo en el componente debido a su carga factorial y pertinencia.

Por otra parte, la tercera dimensión proviene tanto de los factores protectores internos como de los externos; su estructura factorial de cinco reactivos permite organizarla bajo el nombre de "resolución de problemas". Y, por último, los tres reactivos que conforman el cuarto componente pertenecen a la dimensión "factores protectores externos" del cuestionario original, por lo cual su nombre se conserva.

\section{Análisis factorial confirmatorio}

En la muestra aleatoria para el AFC se contó con un total de 261 estudiantes, $52.9 \%$ (138) mujeres y $47.1 \%$ (123) hombres, con una edad promedio de 15.02 años $(\mathrm{DE}=.526)$, de mínimo 14 y máximo 18 . El modelo final mostró una configuración de cuatro factores integrada por 18 reactivos válidos. En la Tabla 4 se muestran los índices de ajuste absoluto de los modelos explorados que surgieron del AFE, siendo el modelo final el que presenta los mejores indicadores.

De igual forma, en la Tabla 5 se muestran los índices de ajuste incremental como referencia comparativa, donde el modelo final exhibe los mejores puntajes.

Finalmente, en la Figura 1 se muestra el modelo de ecuaciones estructurales final del Cuestionario de Resiliencia para Niños y Adolescentes (Revisado).

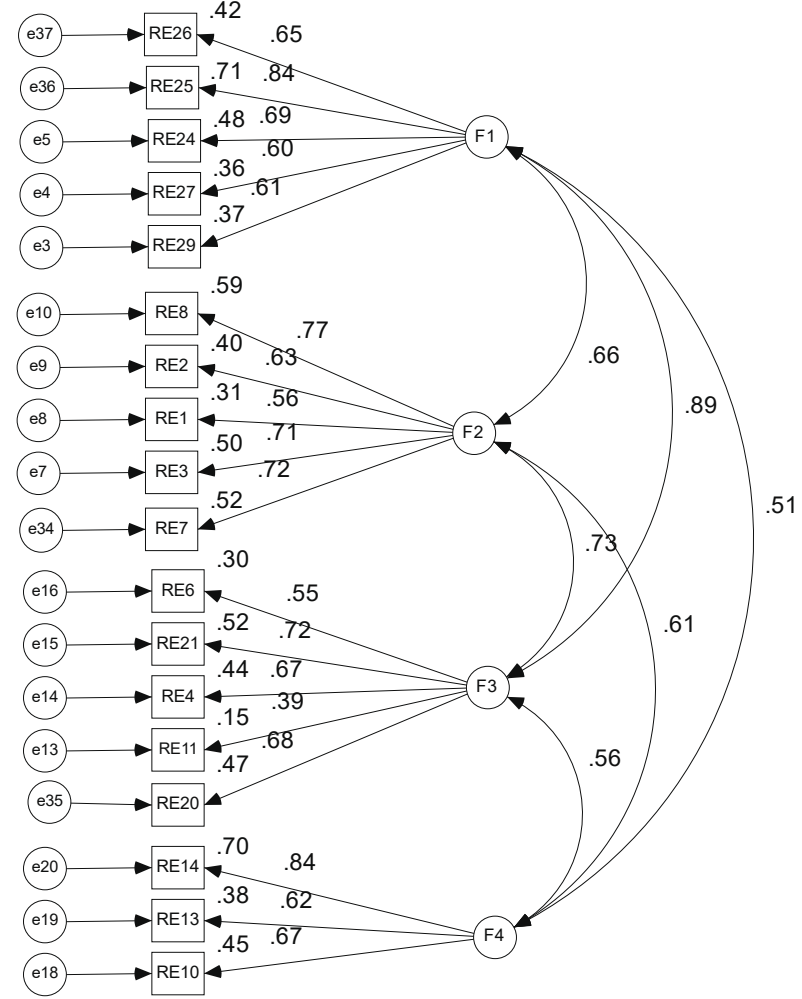

Figura 1. Modelo del análisis factorial confirmatorio del Cuestionario de Resiliencia para Niños y Adolescentes (Revisado). F1 = introspección; F2 = empatía; F3 = resolución de problemas; $\mathrm{F} 4$ = factores protectores externos.

Tabla 4.

Comparación de índices de bondad de ajuste absoluto por modelo

\begin{tabular}{lcccccccc}
\hline & \multicolumn{7}{c}{ Índices de ajuste absoluto } \\
\cline { 2 - 9 } & Factores & CMIN & DF & CMIN/DF & GFI & AGFI & RMR & RMSEA \\
\cline { 2 - 9 } Modelo original & 3 & 2102.587 & 461 & 4.561 & .772 & .739 & .041 & .083 \\
Modelo 1 $^{\mathrm{a}}$ & 5 & 732.644 & 289 & 2.535 & .819 & .78 & .044 & .077 \\
Modelo 2 $^{\mathrm{b}}$ & 5 & 564.784 & 199 & 2.838 & .903 & .876 & .036 & .060 \\
Modelo final $^{\mathrm{c}}$ & 4 & 329.899 & 129 & 2.557 & .931 & .909 & .033 & .055 \\
\hline
\end{tabular}

Nota. ${ }^{\text {a }}$ Eliminando los reactivos $12,15,16,17,31$ y $32 .{ }^{\mathrm{b}}$ Eliminando los reactivos $30,18,9$ y $23 .{ }^{\mathrm{c}}$ Eliminando los reactivos 28 , 5,22 y 19.

Tabla 5.

Comparación de índices de bondad de ajuste incremental por modelo

\begin{tabular}{|c|c|c|c|c|c|c|c|}
\hline \multirow[b]{3}{*}{ Modelo original } & \multicolumn{7}{|c|}{ Índices de ajuste incremental } \\
\hline & Factores & NFI & TLI & CFI & IFI & AIC & $\mathrm{BIC}$ \\
\hline & 3 & .745 & .772 & .788 & .789 & 2236.587 & 2520.838 \\
\hline Modelo $1^{\mathrm{a}}$ & 5 & .784 & .837 & .855 & .857 & 856.644 & 1077.644 \\
\hline Modelo $2^{\text {b }}$ & 5 & .885 & .909 & .922 & .923 & 520.225 & 712.709 \\
\hline Modelo final ${ }^{\mathrm{c}}$ & 4 & .906 & .929 & .94 & .94 & 413.899 & 591.908 \\
\hline
\end{tabular}

Nota. ${ }^{\text {a }}$ Eliminando los reactivos $12,15,16,17,31$ y $32 .{ }^{\mathrm{b}}$ Eliminando los reactivos 30, 18, 9 y 23 . ${ }^{\mathrm{c}}$ Eliminando los reactivos 28 , 5,22 y 19 . 
Análisis de confiabilidad por dimensión

En la Tabla 6 se presentan los índices de confiabilidad general y por subescalas. La escala total alcanzó un nivel excelente de confiabilidad, mientras que las dimensiones "introspección" y "empatía" obtuvieron un nivel de confiabilidad bueno. Por su parte, las dimensiones "resolución de problemas" y "factores protectores externos" lograron un nivel aceptable de confiabilidad.

\section{Validación convergente y divergente}

Puesto que la muestra no presentó normalidad en la distribución, se llevó a cabo un análisis de correlación mediante la prueba rho de Spearman con el propósito de establecer la validez convergente y divergente del cuestionario.

La validación convergente se llevó a cabo al correlacionar las dimensiones del Cuestionario de Resiliencia para Niños y Adolescentes (Revisado) con la Escala de Autoestima de Rosenberg, donde se obtuvieron correlaciones significativas en el nivel .01 para todas las dimensiones. Como puede apreciarse en la Tabla 7, se encontraron correlaciones moderadas para "empatía" y "resolución de problemas", así como para el total de la escala. Por su parte, "introspección" mostró una correlación baja, mientras que la dimensión "factores protectores externos" presentó una correlación muy baja.
La validación divergente se estableció a partir de las correlaciones entre las dimensiones del Cuestionario de Resiliencia para Niños y Adolescentes (Revisado) y el CES-D-R. En la tabla 7 se muestran los resultados, en los que se pueden observar correlaciones significativas en el nivel .01, exceptuando las de "bienestar emocional", donde únicamente se encontró una correlación significativa al nivel de .05 con "resolución de problemas". El factor "empatía" y el total de la resiliencia demostraron correlaciones con un nivel de magnitud moderado, con la salvedad antes descrita. Asimismo, "resolución de problemas" y "afecto positivo" presentaron una correlación moderada, mientras que en el resto se observaron correlaciones muy bajas.

\section{Discusión}

El presente estudio tuvo como objetivo realizar un análisis de las propiedades psicométricas del Cuestionario de Resiliencia para Niños y Adolescentes. El hallazgo principal consiste en ofrecer un modelo modificado con una estructura factorial satisfactoria y un excelente nivel de confiabilidad.

El cuestionario original estaba compuesto por 32 reactivos, los cuales, mediante la reducción factorial, derivaron

Tabla 6.

Análisis de confiabilidad de las dimensiones del Cuestionario de Resiliencia para Niños y Adolescentes (Revisado)

\begin{tabular}{lccc}
\hline \multicolumn{1}{c}{ Dimensión } & Reactivos originales & $\begin{array}{c}\text { Reactivos reordenados en ver- } \\
\text { sión de 18 ítems }\end{array}$ & Alfa de Cronbach \\
\hline Introspección & $26,25,24,27,29$ & $16,15,14,17,18$ & .81 \\
Empatía & $8,2,1,3,7$ & $7,2,1,3,6$ & .80 \\
Resolución de problemas & $6,21,4,11,20$ & $5,13,4,9,12$ & .78 \\
Factores protectores externos & $14,13,10$ & $11,10,8$ & .76 \\
Total & & & .90 \\
\hline
\end{tabular}

Tabla 7.

Matriz de correlaciones entre resiliencia, autoestima y el CES-D-R como medidas de validez convergente y divergente

\begin{tabular}{|c|c|c|c|c|c|}
\hline Variables & Introspección & Empatía & $\begin{array}{c}\text { Resolución de } \\
\text { problemas }\end{array}$ & $\begin{array}{c}\text { Factores protec- } \\
\text { tores externos }\end{array}$ & Resiliencia total \\
\hline Autoestima & $.353 * *$ & $.422 * *$ & $.508 * *$ & $.262 * *$ & $.483 * *$ \\
\hline Afecto deprimido & $-.195 * *$ & $-.358 * *$ & $-.252 * *$ & $-.196 * *$ & $-.317 * *$ \\
\hline Afecto positivo & $.222 * *$ & $.310 * *$ & $.322 * *$ & $.287 * *$ & $.341 * *$ \\
\hline $\begin{array}{l}\text { Inseguridad emocional y prob- } \\
\text { lemas interpersonales }\end{array}$ & $-.205^{* *}$ & $-.406 * *$ & $-.272^{* *}$ & $-.232 * *$ & $-.348 * *$ \\
\hline Somatización & $-.265 * *$ & $-.456 * *$ & $-.284 * *$ & $-.247 * *$ & $-.395 * *$ \\
\hline Bienestar emocional & .058 & -.025 & $.139 *$ & .071 & .070 \\
\hline Actividad retardada & $-.247 * *$ & $-.432 * *$ & $-.250 * *$ & $-.270 * *$ & $-.370 * *$ \\
\hline Malestar depresivo (total) & $-.198 * *$ & $-.407 * *$ & $-.205 * *$ & $-.191 * *$ & $-.318 * *$ \\
\hline
\end{tabular}

Nota. $* \mathrm{p}<.05 * * \mathrm{p}<.01$. 
en 18 ítems con mejores indicadores; además, el modelo consistía en tres dimensiones -factores protectores externos, empatía, y factores protectores internos-, pero tras la revisión, se conservaron los dos primeros factores y se agregaron dos dimensiones emergentes -introspección y resolución de problemas-. Una posible explicación a la aparición de estos factores es la complejidad del constructo, ya que se manifiesta en distintas habilidades; planteamiento afín a lo reportado en la literatura (Luthar, 2015; Rutter, 2012; Walsh, 2004).

La agrupación factorial de las dimensiones obtenidas es coincidente con el modelo teórico original retomado por González-Arratia (2016), donde la dimensión "yo tengo" (apoyo externo) se corresponde con factores protectores externos; la dimensión "yo soy" (fuerza interior) entraría en empatía; mientras que a la dimensión "yo puedo" (capacidades interpersonales y solución de conflictos) corresponderían introspección y resolución de problemas.

Con respecto a la adecuación cultural, es interesante resaltar que, en la versión anglosajona, el modelo se compone de tres factores -"I have", "I am", "I can"- (Grotberg, 1995; 2006), que en el idioma español han sido representados como cuatro, debido al carácter dual del verbo to be, es decir, aparecen las dimensiones "yo tengo", "yo soy", "yo estoy", "yo puedo" (Melillo \& Suarez, 2001). Si bien en el presente estudio se encontraron cuatro dimensiones, estas no se corresponden con tal planteamiento, sino que se apegan más al modelo original, donde -como se mencionó previamente- el factor "yo puedo" integra los factores "introspección" y "resolución de problemas".

Del mismo modo, las dimensiones emergentes son pertinentes a la literatura, ya que, por un lado, coinciden con las elaboraciones teóricas sobre la resiliencia que consideran que el insight -o introspección- implica la capacidad de interpretar situaciones o personas y ajustar el propio comportamiento interpersonal con la manifestación de un conocimiento de sí mismo y de los demás (Grotberg, 1995; Hurtes \& Allen, 2001; Luthar, 2015); y, por otro, la noción de resolución de problemas ya se encontraba incluida en la dimensión original llamada "factores protectores internos" (González-Arratia, 2016), lo cual concuerda con lo reportado previamente (Rutter, 2012).

Adicional a esto, el análisis de las propiedades psicométricas permitió dar soporte a la estructura de cuatro dimensiones diferenciadas, ya que mostró adecuados índices de ajuste tanto absolutos como incrementales, lo que apoya el planteamiento del carácter multidimensional del constructo resiliencia (Hurtes \& Allen, 2001; Luthar, 2015; Walsh, 2004).
Por último, el análisis de validez convergente corroboró empíricamente la estrecha relación entre resiliencia y autoestima, ampliamente reportada en la literatura (GonzálezArratia et al., 2011; Luthar, 2015; Rutter, 2012; Walsh, 2004); a la vez que las pruebas de validez divergente soportaron la evidencia de la asociación negativa entre resiliencia y depresión (Restrepo-Restrepo et al., 2011).

Tal como plantean Hurtes y Allen (2001), para ser útil, un instrumento debe ser simple, fácil de administrar e interpretar, apropiado para la población y relevante para los propósitos de la intervención; y el Cuestionario de Resiliencia para Niños y Adolescentes (Revisado) cubre cada uno de estos criterios, solventando las exigencias de validación, adecuación cultural y contextual (Luthar \& Cushing, 2002; Windle et al., 2011).

Adicional a esto, es importante mencionar tres implicaciones prácticas principales del uso del Cuestionario de Resiliencia para Niños y Adolescentes (Revisado). En primer lugar, la posibilidad de hacer comparaciones entre distintas muestras mediante un instrumento confiable. En segundo, que permite la elaboración de intervenciones que gocen de un sustento empírico para la operacionalización de las dimensiones de la resiliencia. Y, por último, que puede ser utilizado para evaluar y justificar la eficacia de intervenciones y programas a manera de talleres diseñados con el enfoque de resiliencia desde una perspectiva centrada en las fortalezas. Con esto, cabe señalar la importancia de enriquecer las evaluaciones psicométricas con aportaciones psicológicas y contextuales que permitan comprender la complejidad de los procesos resilientes (Belykh, 2018).

La principal contribución del presente artículo a la literatura sobre resiliencia radica en el refinamiento de las propiedades psicométricas del Cuestionario de Resiliencia para Niños y Adolescentes, sobre todo al ser un instrumento desarrollado desde el contexto mexicano. Así, la posibilidad de elaborar intervenciones dirigidas a potenciar la introspección, la empatía, los factores protectores externos y la resolución de problemas a partir de los hallazgos del presente estudio favorecerá la capacidad de los adolescentes de hacer frente a las adversidades normativas e inesperadas de la vida, es decir, que mejorarán su resiliencia.

Finalmente, se reconocen como limitaciones del estudio el tipo de muestreo y el rango de edades, por lo que se recomienda que se lleven a cabo estudios con muestras aleatorizadas en distintas poblaciones, especialmente en niños de educación primaria y secundaria. De igual manera, se sugiere evaluar la estabilidad del instrumento mediante el método test-retest y desarrollar investigaciones de tipo longitudinal. Por último, queda pendiente la baremación del instrumento que permita el uso clínico del mismo. 
302

En conclusión, el Cuestionario de Resiliencia para Niños y Adolescentes (Revisado) cuenta con características psicométricas adecuadas tanto en su estructura factorial y confiabilidad como en su validez convergente y divergente, por lo que se propone como un instrumento útil para la evaluación de la resiliencia en adolescentes mexicanos.

\section{Referencias}

Abad, F., Olea., J., Ponsoda, V., \& García, C. (2011). Medición en ciencias sociales y de la salud. Madrid: Síntesis.

Aguiar, E., \& Acle-Tomasini, G. (2012). Resiliencia, factores de riesgo y protección en adolescentes mayas de Yucatán: elementos para favorecer la adaptación escolar. Acta Colombiana de Psicología, 15(2), 53-64. Recuperado de http://editorial.ucatolica.edu.co/ojsucatolica/revistas ucatolica/index.php/acta-colombiana-psicologia/article/ view $/ 266 /$ pdf $\% 20$ art $\%$ C3\%ADculo $\% 205$

Álvarez, E., González, A., \& Chávez, J. (2017). Conceptos iniciales. En F. González, M. Escoto \& J. Chávez, Estadística aplicada en Psicología y Ciencias de la salud (pp. 13-32). México: Manual Moderno.

Arón, A., \& Milicic, N. (2011). Resiliencia y clima social en el contexto escolar. Psykhe, 4(1), 57-68. Recuperado de http:// www.psykhe.cl/index.php/psykhe/article/view/73/73

Barcelata, B., Durán, C., \& Gómez-Maqueo, E. (2012). Valoración subjetiva de los sucesos de vida estresantes en dos grupos de adolescentes de zonas marginadas. Salud Mental, 35(6), 513-520. Recuperado de http://www.scielo. org.mx/pdf/sm/v35n6/v35n6a9.pdf

Belykh, A. (2018). Resiliencia e inteligencia emocional: conceptos complementarios para empoderar al estudiante. Revista Latinoamericana de Estudios Educativos, 58(1), 255-282. Recuperado de https://drive.google.com/file/d/1 GSpGZZSLNXoVW_6DtIPEVhK9CG1Aj4uL/view

Bromley, E., Johnson, J. G., \& Cohen, P. (2006). Personality strengths in adolescence and decreased risk of developing mental health problems in early adulthood. Comprehensive Psychiatry, 47(4), 317-326. doi: https://doi.org/10.1016/j. comppsych.2005.11.003

Campbell-Sills, L., \& Stein, M. (2007). Psychometric analysis and refinement of the Connor-Davidson Resilience Scale (CD-RISC): Validation of a 10-item measure of resilience. Journal of Traumatic Stress, 20(6), 1019-1028. doi: https:// doi.org/10.1002/jts.20271

Connor, K. M., \& Davidson, J. R. (2003). Development of a new resilience scale: The Connor-Davidson Resilience Scale (CDRISC). Depression and Anxiety, 18(2), 76-82. doi: https://doi.org/10.1002/da.10113

Curran, P. J., West, S. G., \& Finch, J. F. (1996). The robustness of test statistics to nonnormality and specification error in confirmatory factor analysis. Psychological Methods 1(1), 16-29. doi: http://dx.doi.org/10.1037/1082-989X.1.1.16

Donnon, T., \& Hammond, W. (2007). A psychometric assessment of the self-reported youth resiliency: assessing developmental strengths questionnaire. Psychological Reports, 100(3), 963-978. doi: https://doi.org/10.2466/ pr0.100.3.963-978

Escobedo, M., Hernández, J., Estebané, V., \& Martínez, G. (2016). Modelos de ecuaciones estructurales: Características, fases, construcción, aplicación y resultados. Ciencia \& Trabajo, 18(55), 16-22. doi: http://dx.doi. org/10.4067/S0718-24492016000100004

Fiorentino, M. (2008). La construcción de la resiliencia en el mejoramiento de la calidad de vida y la salud. Suma Psicológica, 15(1), 95-114. Recuperado de http://publica ciones.konradlorenz.edu.co/index.php/sumapsi/article/ view/18/4

Friborg, O., Hjemdal, O., Rosenvinge, J. H., \& Martinussen, M. (2001). A new rating scale for adult resilience: What are the central protective resources behind healthy adjustment? International Journal of Methods in Psychiatric Research, 12(2), 65-76. Recuperado de https://www.ncbi.nlm.nih. gov/pubmed/12830300

García-Vesga, M. C., \& Domínguez-de la Ossa, E. (2013). Desarrollo teórico de la Resiliencia y su aplicación en situaciones adversas: Una revisión analítica. Revista Latinoamericana de Ciencias sociales, Niñez y Juventud, 11(1), 63-77. Recuperado de http://www.redalyc.org/articu lo.oa? $\mathrm{id}=77325885001$

Gómez-Azcarate, E., Vera, A., Ávila, M., Musitu, G., Vega, E., \& Dorantes, G. (2014). Resiliencia y felicidad de adolescentes frente a la marginación urbana en México. Psicodebate, 14(1), 45-68. doi: http://dx.doi.org/10.18682/ pd.v14i1.334

González-Arratia, N. I. (2016). Resiliencia y personalidad en niños y adolescente. Cómo desarrollarse en tiempos de Crisis. México: Universidad Autónoma del Estado de México.

González-Arratia, N. I., Reyes, M., Valdéz, J., \& González, S. (2011). Resiliencia, autoestima y personalidad resistente en niños y adolescentes con antecedente de maltrato. Psicumex, 1(1), 51-62. Recuperado de http://www.psicu mex.uson.mx/revistas/articulos/1-r1_art5.pdf

González-Cantero, J., Oropeza, R., Padrós, F., Colunga, C., Montes, R., \& González, V. (2017). Capital psicológico y su relación con el estilo de vida de universitarios mexicanos. Nutrición Hospitalaria, 34(2), 439-443. doi: http://dx.doi. org/10.20960/nh.172

González, N., \& Valdez, J. (2012). Optimismo-pesimismo y resiliencia en adolescentes de una universidad pública. Ciencia Ergo Sum, 19(3), 207-214. Recuperado de http:// www.redalyc.org/articulo.oa?id=10423895002 
González-Forteza, C., Jiménez-Tapia, J., Ramos-Lira, L., \& Wagner, D. (2008). Aplicación de la escala de depresión del Center of Epidemiological Studies en adolescentes de la ciudad de México. Salud Pública de México, 50(4), 292299. Recuperado de http://www.scielo.org.mx/pdf/spm/ v50n4/a07v50n4.pdf

González-Forteza, C., \& Rodríguez, E. (1993). Autoestima y sintomatología depresiva en adolescentes mexicanos: un estudio correlacional por género. Revista da Associação Brasileira de Psiquiatria, 15(2), 59-64. Recuperado de http://bases.bireme.br/cgi-bin/wxislind.exe/iah/ online/?IsisScript $=\mathrm{iah} / \mathrm{iah}$.xis\&src $=$ google $\&$ base $=$ ADOLE C\&lang=p\&nextAction $=$ lnk\&exprSearch $=178145 \&$ indexS earch=ID

Grotberg, E. (1995). A guide to promoting resilience in children: strengthing the human spirit. The International Resilience Project: Bernard Van Leer Foundation. Recuperado de https://bibalex.org/baifa/Attachment/ Documents/115519.pdf

Grotberg, E. (2006). La resiliencia en el mundo de hoy. Cómo superar las adversidades. Barcelona: Gedisa.

Hjemdal, O., Friborg, O., Stiles, T. C., Martinussen, M., \& Rosenvinge, J. (2006). A new scale for adolescents resilience: Grasping the central protective resources behind healthy development. Measurement and Evaluation in Counseling and Development, 39(2), 84-96. doi: https://doi.org/10.10 80/07481756.2006.11909791

Hurtes, K., \&Allen, L. (2001). Measuring resiliency in youth: The resiliency attitudes and skills profile. Therapeutic Recreation Journal, 35(4), 333-347. Recuperado de https://static1. squarespace.com/static/51abe64ee4b0a1344208e98a/t/5 $1 \mathrm{~d} 3 \mathrm{cf1} 15 \mathrm{e} 4 \mathrm{~b} 0 \mathrm{fc} 82 \mathrm{c} 7 \mathrm{~b} 8 \mathrm{ebd} 5 / 1372835605044 / \mathrm{RASP}+\% 2$ 8 Hurtes $+\% 26+$ Allen $\% 2 C+2001 \% 29$.pdf

Jiménez, A., Mondragón, L., \& González-Forteza, C. (2007). Self-esteem, depressive symptomatology, and suicidal ideation in adolescents: results of three studies. Salud Mental, 30(5), 20-26. Recuperado de http://www.redalyc.org/articu lo.oa? $\mathrm{id}=58230504$

Kline, R. (2011). Principles and practice of structural equation modeling. New York: The Guilford Press.

Luthar, S. (2015). Resilience in Development: A Synthesis of Research across Five Decades. En D. Cicchetti \& D. Cohen, Developmental Psychopathology: Volume Three: Risk, Disorder, and Adaptation (pp. 739-795). Wiley: New Yersey.

Luthar, S., \& Cushing, G. (2002). Measurement issues in the empirical study of resilience. En M. Glantz \& J. Johnson (eds). Resilience and development: Positive life adaptations (pp. 129-160). New York: Kluwer Academic.

Masten, A. (2018). Resilience Theory and Research on Children and Families: Past, Present, and Promise. Journal of Family Theory and Review, 10(1), 12-31. doi: https://doi. org/10.1111/jftr.12255

Melillo, A., \& Suarez, E. (2001). Resiliencia. Descubriendo las propias fortalezas. Buenos Aires: Paidós.

Méndez, C., \& Rondón, M. (2012). Introducción al análisis factorial exploratorio. Revista Colombiana de Psiquiatría, 41(1), 197-207. Recuperado de http://www.redalyc.org/ articulo.oa?id=80624093014

Monroy, B., \& Palacios, L. (2011), Resiliencia: ¿Es posible medirla e influir en ella? Salud Mental, 34(3), 237-246. Recuperado de http://www.scielo.org.mx/pdf/sm/v34n3/ v34n3a7.pdf

Oshio, A., Kaneko, H., Nagamine, S., \& Nakaya, M. (2003). Construct validity of the adolescent resilience scale. Psychological Reports, 93(3), 1217-1222. doi: https://doi. org/10.2466/pr0.2003.93.3f.1217

Palomar, J., \& Gómez, N. (2010). Desarrollo de una escala de medición de la Resiliencia con mexicanos. Interdisciplinaria, 27(1), 7-22. Recuperado de http://www. redalyc.org/articulo.oa?id=18014748002

Quiceno, J., Mateus, J., Cardenas, M., Villareal., D., \& Vinaccia, S. (2013). Calidad de vida, resiliencia e ideación suicida en adolescentes víctimas de abuso sexual. Revista de Psicopatología y Psicología Clínica, 18(2), 107-117. doi: https://doi.org/10.5944/rppc.vol.18.num.2.2013.12767

Restrepo-Restrepo, C., Vinaccia, S., \& Quiceno, M. (2011). Resiliencia y depresión: un estudio exploratorio desde la calidad de vida en la adolescencia. Suma Psicológica, 18(2), 41-48. Recuperado de http://publicaciones.konradlorenz. edu.co/index.php/sumapsi/article/view/594/663

Rutter, M. (2012). Resilience: Casual pathways and social ecology. En M. Ungar (ed.), The social ecology of resilience. A handbook of theory and practice (pp. 33-42). New York: Springer.

Ruvalcaba-Romero, N., Gallegos-Guajardo, J., \& VillegasGuinea, D. (2014). Validation of the resilience scale for adolescents (READ) in Mexico. Journal of Behavior, Health \& Social Issues, 6(2), 21-34. Recuperado de http:/www.journals.unam.mx/index.php/jbhsi/article/view/48555/pdf

Ruvalcaba-Romero, N., Gallegos-Guajardo, J., Caballo, V., \& Villegas-Guinea, D. (2016). Prácticas parentales e indicadores de salud mental en adolescentes. Revista Psicología desde el Caribe, 33(3), 223-236. Recuperado de http://www.scielo.org.co/pdf/psdc/v33n3/2011-7485psdc-33-03-00223.pdf

Sánchez-Teruel, D., \& Robles-Bello, M. (2014). Factores protectores que promueven la resiliencia ante el suicidio en adolescentes y jóvenes. Papeles del psicólogo, 35(2), 181-192. Recuperado de http://www.redalyc.org/articulo. oa? id=77832241003 
Schumacker, R., \& Lomax, R. (2010). A beginner's guide to Structural Equation Modeling. New York: Routledge.

Sun, J., \& Stewart, D. (2007). Development of populationbased resilience measures in the primary school setting. Health Education, 7(6), 575-599. doi: https://doi. org/10.1108/09654280710827957

Ungar, M., Liebenberg, L., Boothroyd, R., Kwong, W. M., Lee, T. Y., ... Maknach, A. (2008). The study of youth resilience across cultures: Lessons from a pilot study of measurement development. Research in Human Development, 5(3), 166180. doi: https://doi.org/10.1080/15427600802274019

Villalobos-Galvis, F., Arévalo, C., \& Rojas, F. (2012). Adaptación del Inventario de Resiliencia ante el Suicidio (SRI-25) en adolescentes y jóvenes de Colombia.
Revista Panamericana de Salud Pública, 31(3), 233239. Recuperado de https://www.scielosp.org/pdf/rpsp/ v31n3/08.pdf

Walsh, F. (2004). Resiliencia familiar: estrategias para su fortalecimiento. Buenos Aires: Amorrortu.

Windle, G., Bennet, K., \& Noyes, J. (2011). A methodological review of resilience measurement scales. Health and Quality of Life Outcomes, 9(8), 1-18. doi: https://doi. org/10.1186/1477-7525-9-8 
Anexo

\section{Cuestionario de Resiliencia para Niños y Adolescentes (Revisado)}

A continuación se presenta una serie de preguntas que tienen que ver con tu forma de pensar y actuar ante la vida. Por favor, coloca una (x) en el cuadrado que más se acerque a lo que tú eres, pensando en qué medida crees que te sentiste, actuaste o te comportaste en una situación de crisis o cuando hayas tenido algún problema. Siempre ten presente la situación. No dejes ninguna pregunta sin contestar. Por tu colaboración, muchas gracias.

\begin{tabular}{|c|c|c|c|c|c|c|}
\hline Ítem & Pregunta & $\underset{(5)}{\text { Siempre }}$ & $\begin{array}{l}\text { La may- } \\
\text { oría de las } \\
\text { veces (4) }\end{array}$ & $\begin{array}{l}\text { Indeciso } \\
(3)\end{array}$ & $\begin{array}{l}\text { Algunas } \\
\text { veces (2) }\end{array}$ & Nunca (1) \\
\hline 1 & $\begin{array}{l}\text { Yo soy feliz cuando hago algo bueno para los } \\
\text { demás. }\end{array}$ & & & & & \\
\hline 2 & Yo soy respetuoso de mí mismo y de los demás. & & & & & \\
\hline 3 & Soy agradable con mis familiares. & & & & & \\
\hline 4 & Soy capaz de hacer lo que quiero. & & & & & \\
\hline 5 & Soy inteligente. & & & & & \\
\hline 6 & Soy acomedido y cooperador. & & & & & \\
\hline 7 & Soy amable. & & & & & \\
\hline 8 & $\begin{array}{l}\text { Yo tengo personas que me quieren incondiciona- } \\
\text { lmente. }\end{array}$ & & & & & \\
\hline 9 & $\begin{array}{l}\text { Conmigo hay personas que quieren que aprenda a } \\
\text { desenvolverme solo. }\end{array}$ & & & & & \\
\hline 10 & Cerca de mí hay amigos en quien confiar. & & & & & \\
\hline 11 & $\begin{array}{l}\text { Tengo personas que me quieren a pesar de lo que } \\
\text { sea o haga. }\end{array}$ & & & & & \\
\hline 12 & Soy firme en mis decisiones. & & & & & \\
\hline 13 & $\begin{array}{l}\text { Me siento preparado para resolver mis prob- } \\
\text { lemas. }\end{array}$ & & & & & \\
\hline 14 & Yo puedo controlar mi vida. & & & & & \\
\hline 15 & $\begin{array}{l}\text { Puedo buscar la manera de resolver mis prob- } \\
\text { lemas. }\end{array}$ & & & & & \\
\hline 16 & Puedo imaginar las consecuencias de mis actos. & & & & & \\
\hline 17 & Puedo reconocer lo bueno y lo malo para mi vida. & & & & & \\
\hline 18 & Puedo cambiar cuando me equivoco. & & & & & \\
\hline
\end{tabular}

